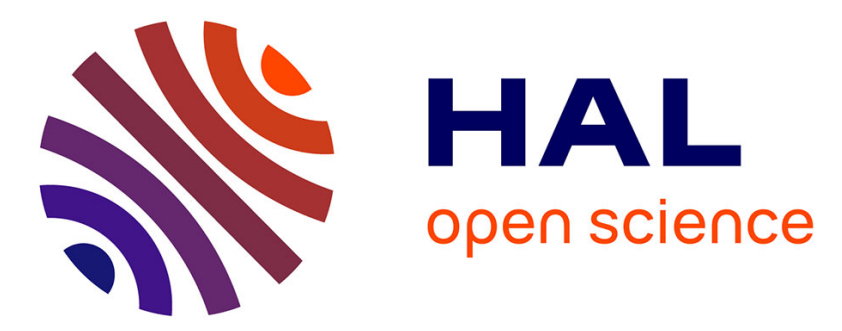

\title{
Orographically generated nonlinear waves in rotating and nonrotating two-layer flow
}

\author{
E. R. Johnson, J. G. Esler, O. J. Rump, Joël Sommeria, G. G. Vilenski
}

\section{To cite this version:}

E. R. Johnson, J. G. Esler, O. J. Rump, Joël Sommeria, G. G. Vilenski. Orographically generated nonlinear waves in rotating and nonrotating two-layer flow. Proceedings of the Royal Society of London. Series A, Mathematical and physical sciences, 2006, 462 (A), pp.3-20. 10.1098/rspa.2005.1550 . hal-00260929

\section{HAL Id: hal-00260929 \\ https://hal.science/hal-00260929}

Submitted on 25 Feb 2020

HAL is a multi-disciplinary open access archive for the deposit and dissemination of scientific research documents, whether they are published or not. The documents may come from teaching and research institutions in France or abroad, or from public or private research centers.
L'archive ouverte pluridisciplinaire HAL, est destinée au dépôt et à la diffusion de documents scientifiques de niveau recherche, publiés ou non, émanant des établissements d'enseignement et de recherche français ou étrangers, des laboratoires publics ou privés. 


\title{
Orographically generated nonlinear waves in rotating and non-rotating two-layer flow
}

\author{
By E. R. Johnson ${ }^{1}, *$, J. G. Esler ${ }^{1}$, O. J. Rump ${ }^{1}$, J. Sommeria ${ }^{2}$ \\ and G. G. Vilenski ${ }^{1}$ \\ ${ }^{1}$ Department of Mathematics, University College London, Gower Street, \\ London, WC1E 6BT UK \\ ${ }^{2}$ LEGI/Coriolis, 21 Avenue des Martyrs, 38000 Grenoble, France
}

This paper reports experimental observations of finite amplitude interfacial waves forced by a surface-mounted obstacle towed through a two-layer fluid both when the fluid is otherwise at rest and when the fluid is otherwise rotating as a solid body. The experimental apparatus is sufficiently wide so that sidewall effects are negligible even in near-critical flow when the towing speed is close to the interfacial long-wave speed and the transverse extent of the forced wavefield is large. The observations are modelled by a simple forced Benjamin-Davis-Acrivos equation and comparison between integrations of both linear and nonlinear problems shows the fundamental nonlinearity of the nearcritical flow patterns. In both the experiments and integrations rotation strongly confines the wavefield to extend laterally over distances only of order of the Rossby radius and also introduces finite-amplitude sharply pointed lee waves in supercritical flow.

Keywords: nonlinear waves; orography; two-layer rotating flow; forced rotating BDA equation

\section{Introduction}

Among the many observations of cloud patterns near orography, some stand out as showing stationary disturbances upwind of the orography. Johnson \& Vilenski (2004) (simply 'JV' here) reproduce a NASA photograph of Guadalupe Island, Baja California taken during the Gemini-V flight and described by Stevenson (1969). A low layer of stratocumulus cloud is moving at 6-10 knots past the island whose peaks reach $4500 \mathrm{ft}$ and thereby project through, and interfere with, the cloud layer. Stevenson (1969) notes that a 'shock or bow' wave spreads from the north end of the island, 'similar to waves formed by a ship moving through water', and further observes that as these cloud features were photographed during four Gemini missions they must be considered climatic features of the Guadalupe marine atmosphere, whose fluid dynamic details must be investigated for proper analyses of atmospheric and ocean flows. Burk \& Haack (1999) describe similar wave clouds extending away from the Monterey-Big Sur coastline of central California, noting that it is the orographic forcing and stationary appearance in geostationary operational environment satellite * Author for correspondence (e.johnson@ucl.ac.uk). 
(GOES) image loops that set these waves apart from propagating wave phenomena. They present a schematic description based on isolated single attached and detached shocks forced by obstacles in supercritical flows. Tjernstrom \& Grisogono (2000) and Soderberg \& Tjernstrom (2001) also discuss flow in the atmospheric boundary layer around points and capes in terms of the criticality of the flow, following Samelson (1992) and Samelson \& Lentz (1994) who treat the shallow marine atmospheric boundary layer under a low-level inversion along the Californian coast as supercritical flow with a sidewall. Jiang \& Smith (2000) model these phenomena theoretically in the context of shallow water flow over isolated orography, noting that a shallow water model can capture such atmospheric flow phenomena as long wakes in the lee of obstacles, quasi-steady vortices and vortex shedding, and observing that with realistic parameters a single-layer model as a representation of stratified air flow has even made some reasonable quantitative predictions of vortex shedding period and wake length (Schär \& Smith 1993). Because of the direct analogy between the shallow water equations and the compressible two-dimensional Euler equations, these analyses lead to flow patterns with isolated attached and detached shocks as in the schematic descriptions of Burk \& Haack (1999) and Jiang \& Smith (2000). However, the GOES observations and nonhydrostatic numerical computations in Burk \& Haack (1999) show a wave pattern that does not necessarily consist of an isolated cloud line but can consist of an initial line followed by a set of parallel lines of cloud. Figure 1 shows a closer view of Guadalupe Island from a more recent (11 July 2001) NASA photograph. The parabolic bow wave is again clearly visible, bearing out Stevenson's (1969) observation that it is a climatic feature, but behind the initial bow wave a series of alternate crests and troughs also appears.

JV note that extending the shallow water equations to include weak nonhydrostatic effects causes the single isolated shocks of the ship-wave analogies of Stevenson (1969), Burk \& Haack (1999) and Jiang \& Smith (2000) to split into multiple parallel crests similar to those observed. JV consider near-critical flow, where the oncoming flow speed is close to the dominant free-wave speed of the flow, in the limit where wave steepening due to weak nonlinearity is balanced by weak dispersion due to non-hydrostatic effects. For one-dimensional flow over ridges this leads to the forced Korteweg-de Vries (fKdV) equation (Grimshaw \& Smyth 1986) and in two-dimensional flow over isolated orography to the forced two-dimensional KdV or forced Kadomtsev-Petviashvili (fKP) equation (Kadomtsev \& Petviashvili 1970; Akylas 1994). Solutions reproduce many of the features seen in numerical integrations of the fully nonlinear shallow-water equations of Jiang \& Smith (2000) and also give predictions of the drag exerted on the flow by the orography, perhaps one of the most important physical quantities required from local modelling to parameterize orography in large-scale models.

Li et al. (2004) report upstream propagating solitary waves identified both from synthetic aperture radar and moderate resolution imaging spectroradiometer images near St Lawrence island in the Bering Sea. To model these observations numerically they treat the waves as one-dimensional and approximate the density profile as a two-layer fluid. The governing equation then becomes the forced extended $\mathrm{KdV}$ (feKdV) equation where, for sufficiently large amplitude waves or particular ratios of densities and depths, cubic 


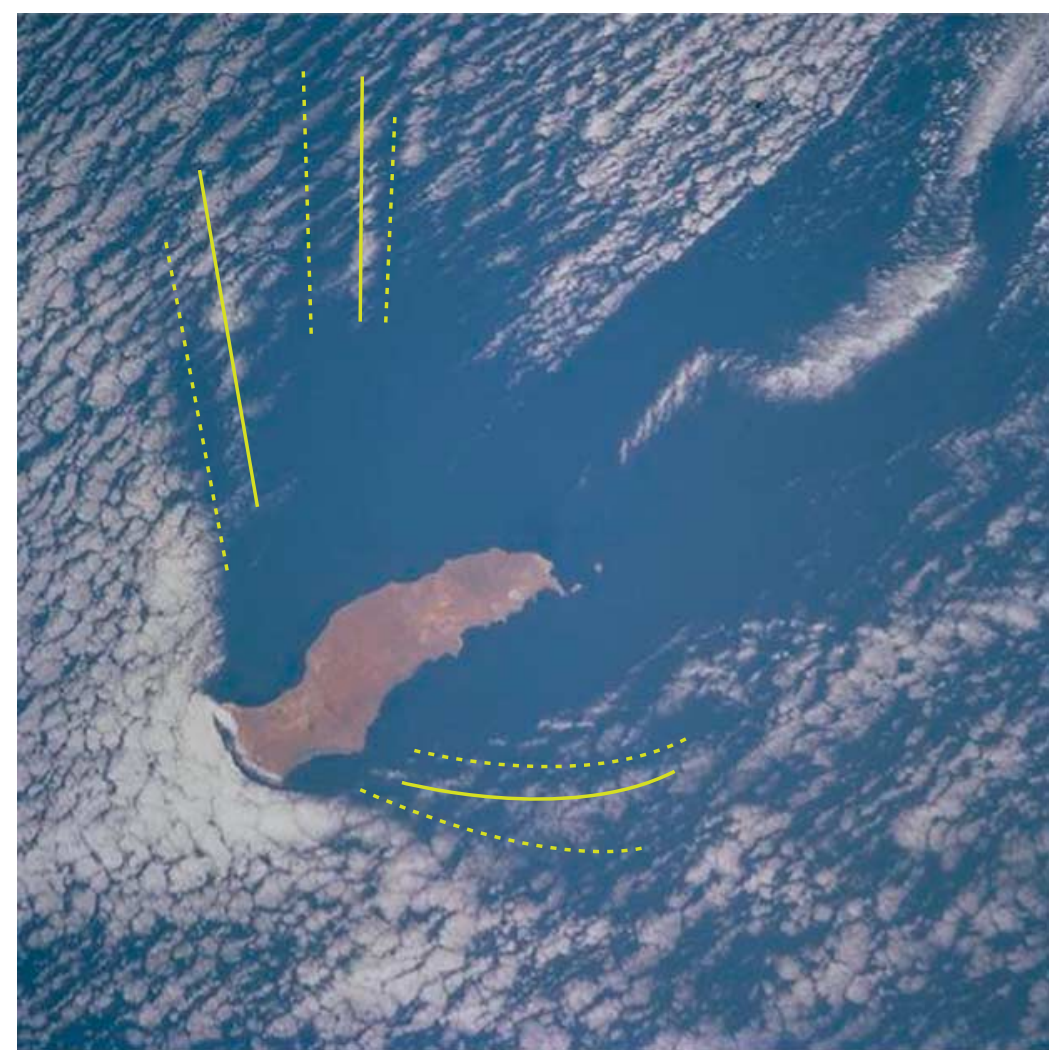

Figure 1. A wavefield above Guadalupe Island, Baja California with crests (solid lines) and troughs (dashed lines) indicated.

nonlinearities can contribute to the motion in addition to the usual quadratic nonlinearities of the KdV (Melville \& Helfrich 1987; Grimshaw et al. 2002). Johnson \& Vilenski (2005) note the modification to these flow patterns when the forcing is more localized so the flow is weakly two-dimensional, governed by the forced extended KP (feKP) equation. Similar solitary waves advancing away from the front of an obstacle have been generated and observed in the laboratory by Maxworthy et al. (1984). In both the cases described by Maxworthy et al. (1984) and $\mathrm{Li}$ et al. (2004), the flows are significantly subcritical and waves advance unsteadily upstream. The unsteady development of forced twodimensional solitary waves is described in Lee \& Grimshaw (1990) and Li \& Sclavounos (2002). It is the purpose of this paper to investigate experimentally, and model simply, nearly steady near-critical flow patterns, both weakly subcritical and weakly supercritical, to provide a possible mechanism for the observed steady flow patterns noted earlier and shown in figure 1.

Before applying analyses like these to planetary flows it is necessary to estimate the effect of the Earth's rotation on the flow. Ostrovsky (1978) considers weak rotation in unforced one-dimensional flows and this, together with later work on one-dimensional flows and some two-dimensional unforced flowsgoverned by the rotating KP (rKP) equation-are described in detail in the reviews of Akylas (1994) and Grimshaw et al. (1998b). In particular, Grimshaw 
et al. (1998b) point out that rotational effects are important when they become comparable to non-hydrostatic dispersion and that in oceanic flows this occurs for motions with scales of the order of a few hundreds of kilometres in the open ocean and only a few tens of kilometres in shallow seas. Vilenski \& Johnson (2004) consider an obstacle advancing at approximately critical speed into an otherwise quiescent rotating fluid, a set-up chosen for the possibility of verifying predicted flow fields against relatively straightforward towing tank experiments and because the natural division of non-rotating flows into subcritical, critical and supercritical flows remains. The flow is governed by the forced rKP (frKP) equation. They describe weakly nonlinear supercritical solutions showing how steady oblique solitary waves, unattenuated in non-rotating flow, decay with distance from the obstacle and how they can be interpreted in terms of the temporal decay of solitary waves in the one-dimensional problem described by Grimshaw et al. (1998a). These effects appear here in both the experimental and model results.

The fKP equation describes not only the vertical displacement of the freesurface of a near-critical shallow flow but also, as shown for the fKdV by Grimshaw \& Smyth (1986), the horizontal and temporal variation of the amplitude of a near-critical vertical mode in a continuously stratified flow of finite depth. Grimshaw \& Smyth (1986) show that, for oncoming flows with speeds near that of the fundamental linear mode, the vertical structure of the mode influences the governing equation only through the coefficients in the equation, determined by integrals of the vertical structure. Computed patterns, like those in JV, thus depend only weakly on the details of the vertical structure of the linear wave. This weak dependence of the horizontal patterns on vertical linear wave structure offers a possible explanation of the qualitative accuracy of the description of near-critical flows by the shallow water equations even when the vertical structure of the fundamental linear wave differs from the two-layer reduced gravity model. Such differences in vertical wave structure have been remarked on in models of coastally trapped waves on a inversion within uniformly stratified layers by Samelson (1999) and Durran (2000), although the vertical step orography in their examples leads to singular pressure fields (Schmidt \& Johnson 1993b) and differences near continuous orography (following, for example, Schmidt \& Johnson 1993a) could be smaller.

Rottman \& Einaudi (1993) review weakly nonlinear theory for the propagation of one-dimensional solitary waves in the atmosphere. They distinguish between two typical scenarios. In the first scenario the waves propagate within a strongly stratified waveguide with relatively weak stratification elsewhere. Jiang \& Smith (2000) note that in the marine atmosphere the top of the boundary layer often acts as this waveguide. In the second scenario the stratification is relatively uniform throughout most of the depth of the fluid, and in the atmospheric case the waves then occupy most of the troposphere with the tropopause and strongly stable stratosphere acting as an upper boundary, to give an effectively finitedepth flow. The first scenario leads to Benjamin-Davis-Acrivos (BDA) (Benjamin 1967; Davis \& Acrivos 1967; Ono 1975) theory and the second to KdV theory. Hence Rottman \& Einaudi (1993) note that in the atmosphere BDA theory applies mostly to low-level solitary waves, where waves propagate in a low-level stable layer topped by a very weakly stratified layer, whereas the KdV theory generally applies to deeper waves that occupy most of the troposphere. 


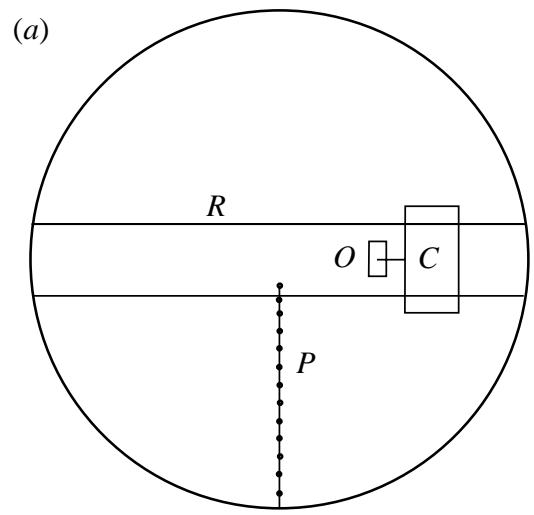

(b)

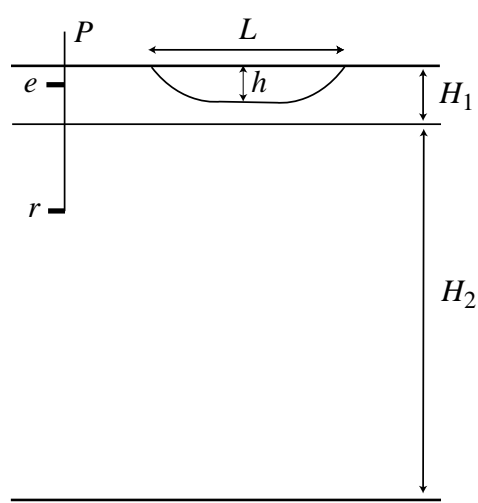

Figure 2. The experimental set-up. (a) The top view. (b) A vertical cut. The obstacle $O$ is moved along the upper layer by the carriage $C$ which rides along rigid rails $R$ sufficiently far above the surface to clear the spar supporting the interfacial probes $P$. Each probe consists of an ultrasonic emitter/receiver $e$ above the interface and a reflector $r$ below the interface.

They point out that the atmosphere often produces layers of nearly neutrally stratified air that are sufficiently deep to provide effective trapping of solitary waves (the BDA case), but rarely produces a sufficiently large increase in buoyancy frequency to make a good reflector and effectively cap a stratified layer (the KdV case). They suggest that the KdV may, however, be relevant when there is sufficient vertical shear in the oncoming flow to reflect vertically propagating internal waves. As the lower layer in the experiments described below is deep and neutrally stable and the effective oncoming flow is unsheared, the experiments relate most closely to the BDA case and are modelled below in terms of a forced, rotating, weakly two-dimensional BDA (fr2dBDA) equation.

Section 2 describes the experimental set-up of a two-layer fluid in a large rotating tank where a thin upper layer occupies $10 \%$ of the total fluid depth. Section 3 interprets the experimental observations of interface displacement in terms of solutions the fr2dBDA equation and $\S 4$ gives brief conclusions.

\section{Experimental set-up}

The experiments were performed in the large rotating tank at the LEGI-Coriolis facility in Grenoble, the same facility as used in the experiments of Maxworthy et al. (1984). The present set of experiments differed from those of Maxworthy et al. (1984) in that here the obstacle ( $O$ in figures 2 and 3) was mounted on a carriage $C$ whose speed could be accurately controlled so the obstacle could be moved along the water surface at constant speed. In the experiments of Maxworthy et al. (1984), the obstacle was bottom-mounted and moved only a fixed distance before being brought to rest; so interfacial solitary waves propagated away in front of the obstacle. One of the great advantages of the Coriolis set-up is the large width of the tank. The wavefield forced by obstacles towed at near-critical speeds spreads laterally over significant distances and in narrower domains wave reflection from sidewalls soon dominates the entire wavefield (Ertekin et al. 1986). In the present experiments wall reflections do not 


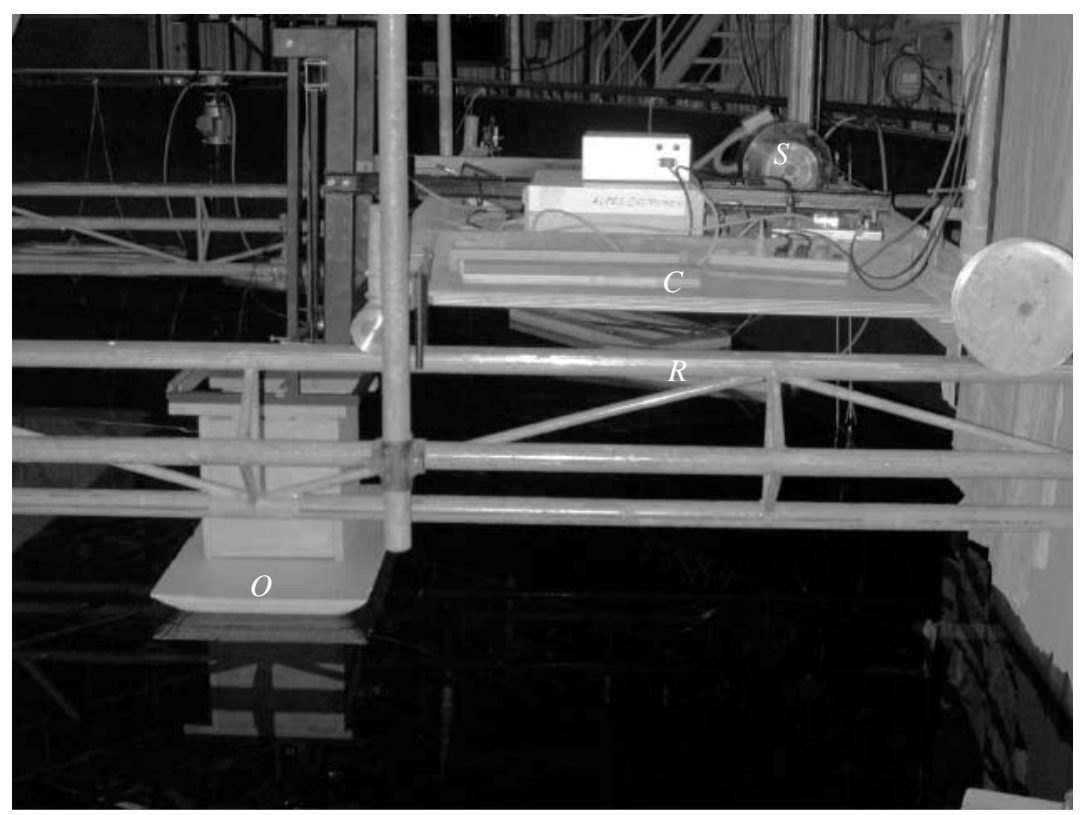

Figure 3. A side photograph of the experimental apparatus. The oblong obstacle $O$ used in the set of experiments described in $\S \S 2$ and 3 has dimensions $50 \mathrm{~cm} \times 100 \mathrm{~cm}$. The speed at which the carriage $C$ is moved along the rails $R$ is accurately controlled by the stepper motor $S$.
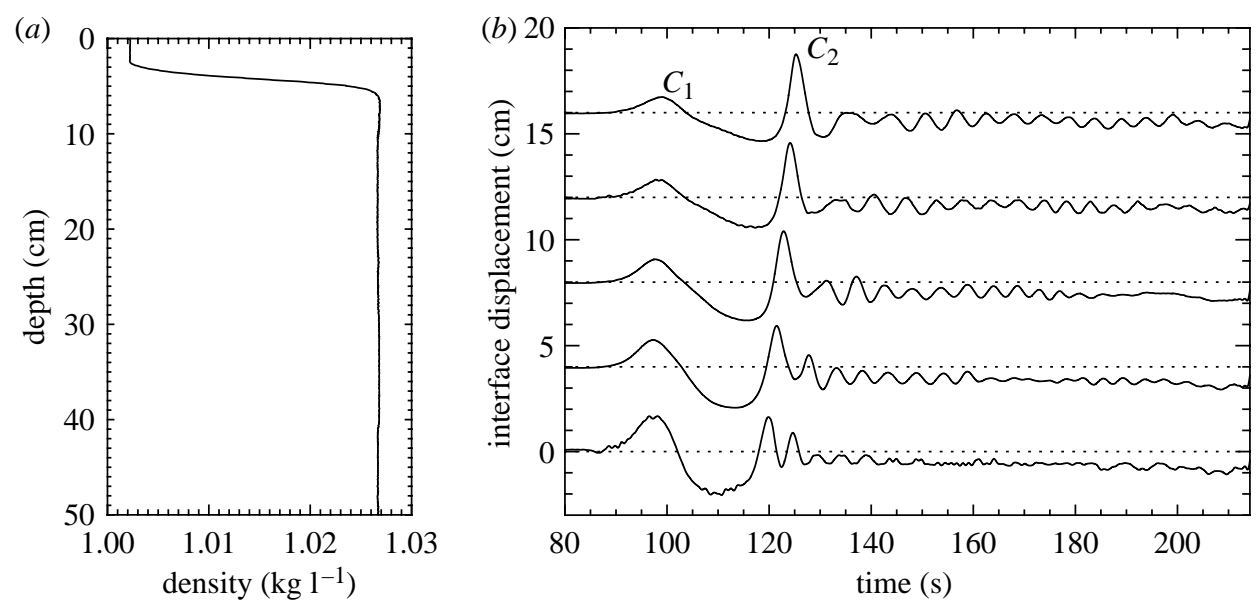

Figure 4. (a) A typical density profile showing the particularly sharp interface of the rotating experiments. (b) The time-series of interface displacements from the innermost five probes (in order from the closest at the bottom, with successive traces displaced upwards by $4 \mathrm{~cm}$ to avoid overlap) for figure 5 for rotating flow with towing speed $U=10 \mathrm{~cm} \mathrm{~s}^{-1}$. The crest of the bow wave is marked $C_{1}$ and the more prominent sharp-crested lee wave is marked $C_{2}$.

affect the flow until long after the dominant flow pattern had passed the measurement line. The present experiments differed also from those of Maxworthy et al. (1984), which used two thin fluid layers, in having a layer of thickness $H_{1}=6 \mathrm{~cm}$ floating on a much deeper lower layer of thickness $H_{2}=54 \mathrm{~cm}$ 

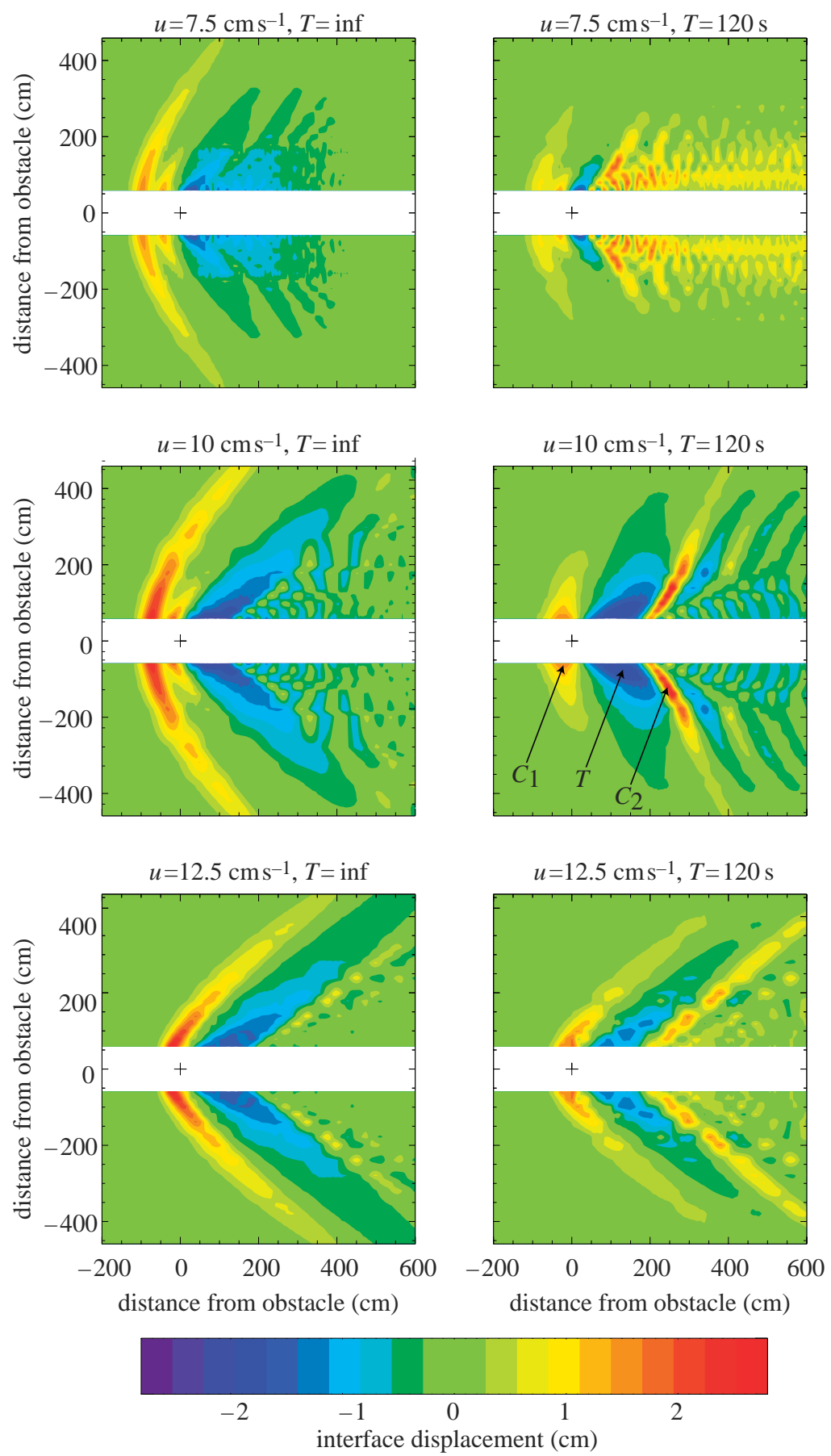

Figure 5. Observed experimental interface elevations for an oblong obstacle at towing speeds $U=7.5,10$, and $12.5 \mathrm{~cm} \mathrm{~s}^{-1}$. Left column: non-rotating. Right column: rotating with period $T=120 \mathrm{~s}\left(f=0.105 \mathrm{~s}^{-1}\right)$, giving a Rossby radius of approximately $1.0 \mathrm{~m}$. The centre of the obstacle is indicated by the ' + ' at the origin. As in figure $4 b, C_{1}$ marks the crest of the bow wave, $C_{2}$ the more prominent sharp-crested lee wave and $T$, the long deep trough between them. 
(figure $4 a$ ). The aim was to give a system to model, when inverted, an atmospheric flow consisting of a thin lower layer below a much deeper upper layer, following Rottman \& Einaudi (1993) and Jiang \& Smith (2000) who note that the marine atmosphere typically has a two-layer stratification profile, with the marine atmospheric boundary layer forming a lower layer of 1-2 km beneath free tropospheric air of depth $8-10 \mathrm{~km}$. The value of inverting the experimental system is twofold: first, no problems arise due to bottom boundary layer effects at the obstacle and, second, the upper layer can be readily refreshed after each experiment with fresh water. The density difference was $2.5 \%$, corresponding to interfacial long wavespeeds $c_{0}=\left(g^{\prime} H_{1}\right)^{1 / 2}$ of $10 \mathrm{~cm} \mathrm{~s}^{-1}$. The obstacle was moved across a diameter of the tank at a constant speed $U$ in the range $5-30 \mathrm{~cm} \mathrm{~s}^{-1}$, allowing the transition from subcritical to supercritical regimes to be explored in all cases. At these speeds the Froude number associated with the free surface is extremely small and consequently the upper surface acts as a rigid lid. During the experiments no surface waves were observed. In addition to non-rotating runs, the Coriolis facility allowed experiments with tank rotation periods of $T=60,90$ and $120 \mathrm{~s}$.

Interface displacements were measured by a set of 20 acoustic probes $(P$ in figure 2) spaced $20 \mathrm{~cm}$ apart along a spar transverse to and to one side of the obstacle motion. The probes consisted of an ultrasonic pulse emitter ( $e$ in figure 2 ), positioned above the interface, and a reflector (a small metallic plate, $r$ in figure 2), positioned below it. The piezo-electric emitter was also used as a receiver, and the return time of the ultrasonic pulses measured. As the speed of sound is different in both layers, this return time varies linearly with interface displacement. The precision of measurement was $0.1 \mathrm{~mm}$. The probe calibration was checked before each experiment by applying a vertical translation of $1 \mathrm{~cm}$ to the whole spar of probes, using a stepping motor. Similar interfacial probes have been used previously by Ramirez \& Renouard (1998). Figure $4 b$ gives time-series from a typical experimental run. Runs were performed in both directions to verify that the flow was symmetric about the centreline. Individual runs with the same parameters showed a high degree of reproducibility and runs with the same parameters in opposite directions showed good agreement: at the rotation rates here the observed wavefields away from the direct wake region were symmetric about the centreline. Thus the interface elevations in figure 5 are shown with data reflected about the centreline to represent the whole wavefield. The absence of data in the band near the centreline is due to the inner limit placed on the probe line to allow the obstacle to pass without obstruction. The gap between the innermost probe and the edge of the widest obstacle was approximately $9 \mathrm{~cm}$. Time-series of interface displacements from the probes like those in figure $4 b$ were converted into the spatial plots of figure 5 by assuming that the flow pattern was stationary relative to the obstacle. The validity of this assumption is discussed briefly in $\S 4$.

Typical runs in the tank, of diameter $13 \mathrm{~m}$, took approximately $2 \mathrm{~min}$. Data from the probes were stored in raw form, a simple program converted this to displacements in centimetres and a further program plotted these in the form of a frame of figure 5 . Thus within a few minutes of the end of a run it was possible to examine the generated wavefield. In practice, it was necessary to wait about 20 min for the wavefield to decay after each run and so it was possible to perform over 18 runs a day with a given stratification and rotation speed while varying 
obstacle height and towing speed. The disruption to the basic stratification was minimal over one day but over a three-day period the interface thickened altering the interfacial wavespeed and making it more difficult to estimate the fractional depth of the upper layer occupied by the obstacle. At this stage the tank was drained, the two layers mixed, sufficient salt added to return the density to $2.5 \%$ above freshwater, this mixed fluid used to form the lower layer and freshwater added to form a new upper layer.

Various obstacle shapes were used in the experiments, including axisymmetric Gaussian obstacles of different volumes and the flat obstacle of figure 3. It was found that the wavefield generated by symmetric obstacles was determined almost entirely by the volume of the obstacle and was uninfluenced by the obstacle profile. Observations are thus reported below solely for the flat obstacle.

\section{Interpretation of the experimental results}

\section{(a) A model equation for forced, rotating, near-critical internal waves}

As the lower layer is neutrally stratified and 10 times deeper than the upper layer, it is treated here as effectively infinitely deep so the appropriate model equation can be expected to belong to the BDA family. Following the derivation in Grimshaw (1985) and Johnson (1997), and including modifications for twolayer flow (e.g. Ono 1975), and for rotation (Ostrovsky 1978), gives the forced, rotating, weakly two-dimensional BDA equation (fr2dBDA),

$$
2 \eta_{0 \zeta \tau}+2 \Gamma \eta_{0 \zeta \zeta}+\frac{3}{2}\left(\eta_{0}^{2}\right)_{\zeta \zeta}+\mathcal{H}\left[\eta_{0}\right]_{\zeta \zeta \zeta}-\nu^{2} \eta_{0}+\mathcal{A}^{2} \eta_{0 Y Y}=-M B_{\zeta \zeta},
$$

for the evolution of the leading order interface displacement $\eta_{0}$. Here $\mathcal{H}$ is the Hilbert transform

$$
\mathcal{H}[f(x)]=\frac{1}{\pi} P V \int_{-\infty}^{\infty} \frac{f\left(x^{\prime}\right)}{x^{\prime}-x} \mathrm{~d} x^{\prime},
$$

where PV denotes the Cauchy principal value of the integral. The onedimensional form of equation (3.1) has been used previously by Matsuno (1995) to describe the collision of a soliton with a ridge.

The derivation of equation (3.1) follows from expansion in the small parameter $\delta=H_{1} / L \ll 1$, and it follows that $\zeta=\delta(x-U t) / H_{1}$ is a spatial coordinate fixed in the frame of the obstacle which is moving at speed $U, Y=\delta^{3 / 2} \mathcal{A} y / H_{1}$ is a stretched $y$-coordinate and $\tau=\delta^{2} c_{0} t / H_{1}$ is a 'slow' time variable. At leading order $\eta_{0}$ is related to the true interface displacement $\eta$ via $\eta=\delta \eta_{0}$ and $B$ is a nondimensionalized obstacle height with a maximum value of unity. There are four non-dimensional parameters $\Gamma, \mathcal{A}, \nu$ and $M$ associated with equation (3.1). They can be identified with criticality $(\Gamma<0$ indicates a super-critical obstacle tow speed), obstacle anisotropy, rotation and nonlinearity, respectively. These nondimensional parameters are directly related to the experimental parameters as follows:

$$
\Gamma=\frac{c_{0}-U}{c_{0}} \delta^{-1}, \quad \mathcal{A}=\frac{L}{L_{y}} \delta^{-1 / 2}, \quad \nu=\frac{f H_{1}}{c_{0}} \delta^{-3 / 2}, \quad M=\frac{h}{H_{1}} \delta^{-2} .
$$



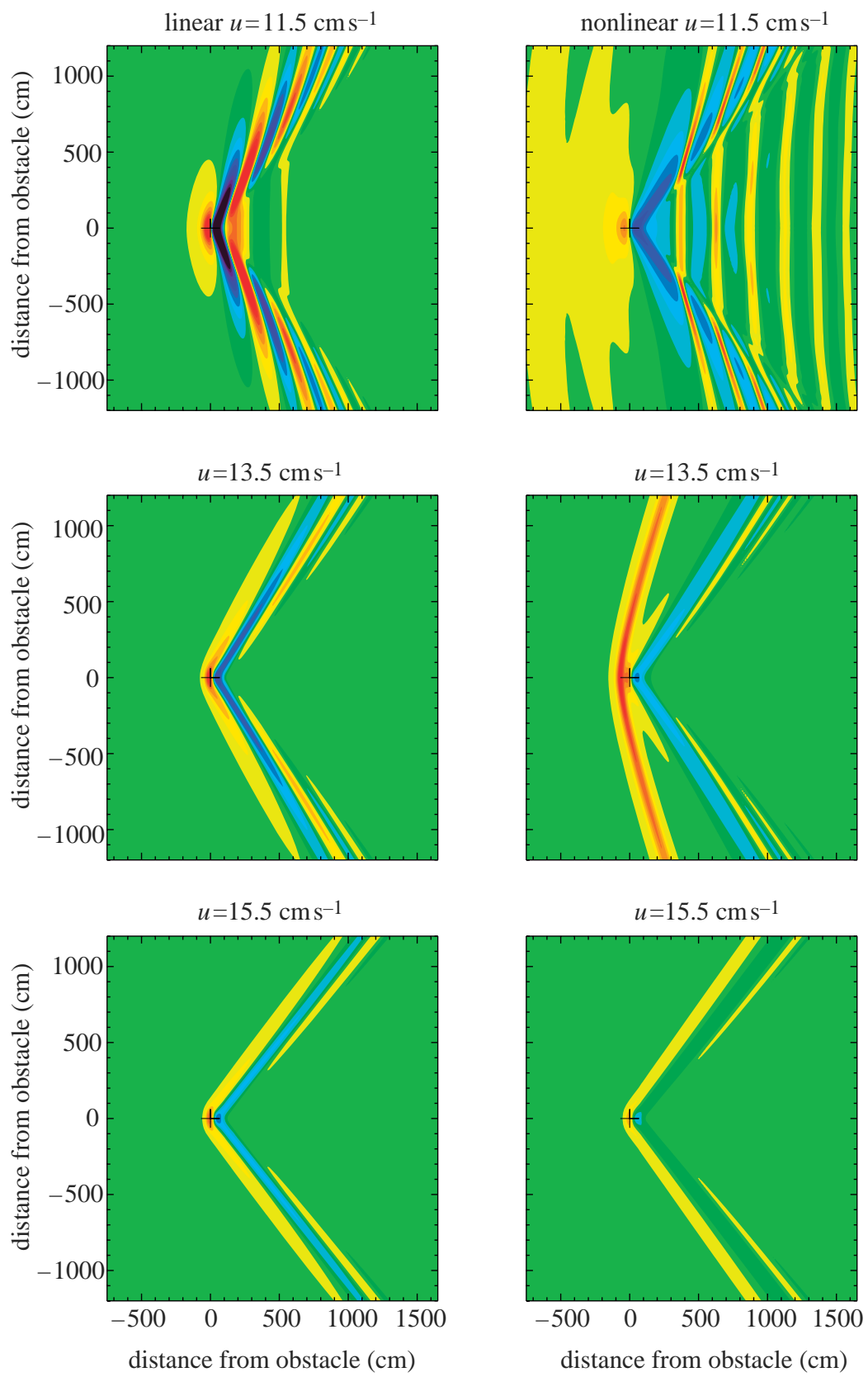

distance from obstacle $(\mathrm{cm})$

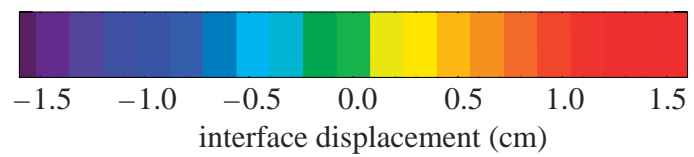

Figure 6. Linear and nonlinear non-rotating $(\nu=0)$ f2dBDA steady states corresponding to obstacle height $h=3 \mathrm{~cm}(M=12.5)$ and towing speeds $U=11.5,13.5$ and $15.5 \mathrm{~cm} \mathrm{~s}^{-1}(\Gamma=0.208,-0.625$, $-1.458)$. 

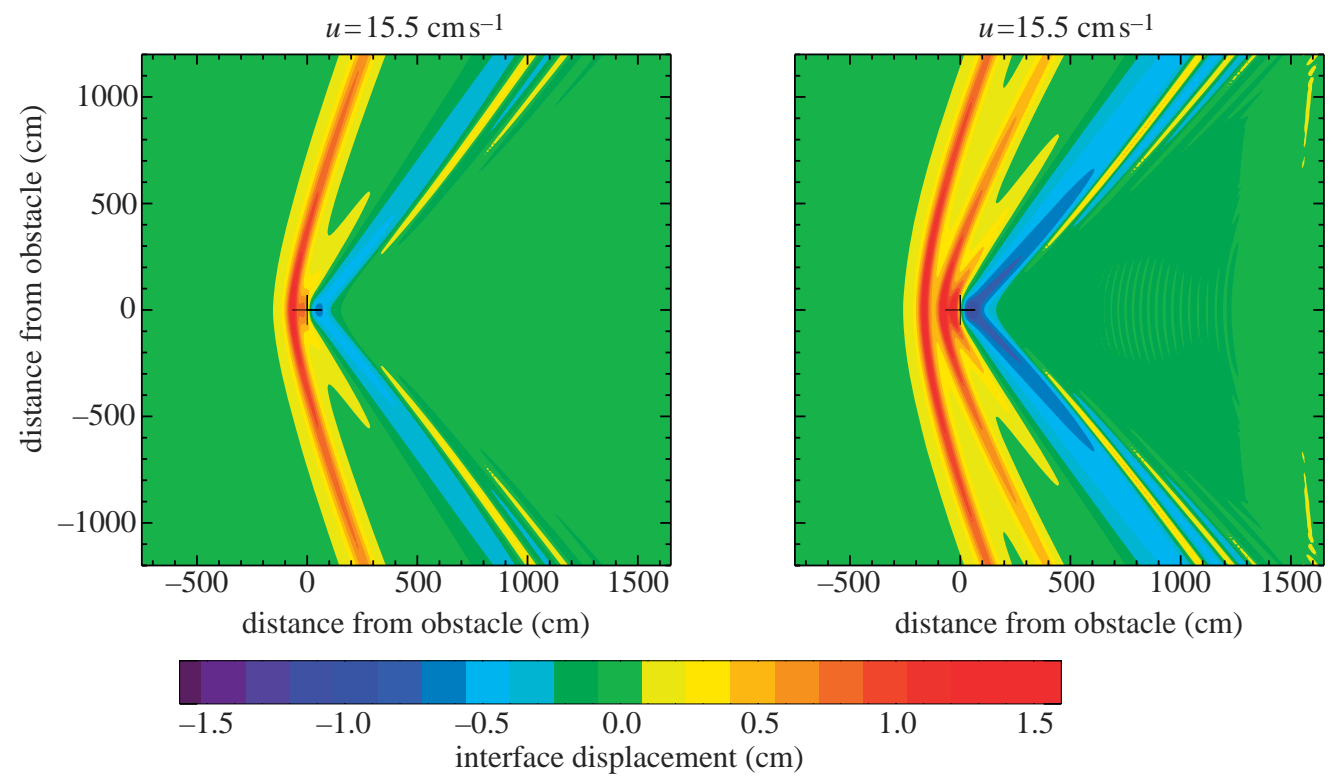

Figure 7. Non-rotating f2dBDA steady states for $U=13.5(\Gamma=-0.625)$ at nonlinearities corresponding to: Left panel: $h=3 \mathrm{~cm}(M=12.5)$. Right panel: $h=5 \mathrm{~cm}(M=20.83)$.

Recall that $h$ is the maximum height of the obstacle; $L_{y}$, the obstacle length scale in the $y$-direction; $c_{0}=\left(g^{\prime} H_{1}\right)^{1 / 2}$, the speed of long interfacial gravity waves, with $g^{\prime}$ the reduced gravity; and $f$, the Coriolis parameter. A balance of the final two terms on the left side of equation (3.1) shows that solutions can be expected to decay away from the axis over distances of the order of the Rossby radius, $c_{0} / f$. It is noted below that this decay is more evident in the experimental observations that in either the linear or nonlinear solutions of equation (3.1).

\section{(b) Numerical calculations}

In order to differentiate between the separate roles of criticality, rotation and nonlinearity in determining the wave pattern generated by the obstacle, equation (3.1) was integrated numerically on a workstation. Equation (3.1) is a model equation for the experimental problem and the results should be interpreted as such, rather than as a direct numerical simulations of the experiments. Nevertheless, in order to ensure that the parameter regimes most relevant to the experiments are explored, the model equation parameters $\Gamma, \mathcal{A}, \nu$ and $M$ are calculated in each case from the corresponding experimental parameters using equation (3.2).

Equation (3.1) was solved in a doubly periodic domain, with dimensions $120 L \times 120 L_{y}$ in terms of the obstacle length scales $L$ and $L_{y}$. The integrations continue until the interface height is found to be steady in a subdomain of dimensions $40 L \times 40 L_{y}$ centred on the obstacle. The discretization is pseudospectral, and a resolution of $512 \times 512$ Fourier modes was found to be adequate to resolve the wave patterns in the parameter regime of interest. (Higher resolution was found necessary when nonlinearity dominated over dispersion, i.e. at large 
$M$, small $|\nu|,|\Gamma|$.) Time-stepping in the model proceeds according to the predictor-corrector method with time-step $\Delta \tau=0.1$.

The obstacle shape $B$ is taken in the numerical integrations to be the Gaussian

$$
B(\zeta, Y)=\exp \left\{-\left(\zeta^{2}+Y^{2}\right)\right\} .
$$

This shape is numerically convenient as it has little signature at the very high wavenumbers that can affect the time-stepping in the numerical model but are absent from the experimental observations due to weak viscous effects. The length scales of the obstacle are chosen as $L=30 \mathrm{~cm}$, and $L_{y}=60 \mathrm{~cm}$ to give the same volume and aspect ratio as the experimental obstacle. In all the numerical integrations described $\Delta \rho / \rho=2.5 \%$, and $H_{1}=6 \mathrm{~cm}$, so that $\mathcal{A}=1.12$ and, for the purposes of determining the other non-dimensional parameters using equation (3.2), $\delta=1 / 5$ and $c_{0}=12 \mathrm{~cm} \mathrm{~s}^{-1}$. The parameters $\Gamma, \nu$ and $M$ vary as the tow speed $U$, the rotation rate $f$ and the obstacle height $h$, respectively, are varied. To compare with the nonlinear solutions, linear calculations of the steady-state wake pattern were also obtained. For each Fourier component, equation (3.1) was solved with the nonlinear terms absent, and with the operator $\partial / \partial t$ replaced by a scalar factor $\varepsilon=0.04$. The use of a positive scalar factor ensures the correct causality in the solution for the obstacle wake (Lighthill 1965), and the limit $\varepsilon \rightarrow 0$ corresponds to the exact linear solution in an infinite domain. In practice a finite value of $\varepsilon$ is necessary to restrict the scale of the wake to within that of the finite domain.

The obstacle used in the experiments had a width of the order of the total fluid depth and so, to determine the importance of the particular model equation chosen, integrations were also performed using the frKP equation following Vilenski \& Johnson (2004) and the feKP equation following Johnson \& Vilenski (2005), using linear wave parameters from both the two-layer model and computed from the measured experimental density profiles. With parameter values derived from the experiments, all models showed the same qualitative behaviour, and so only integrations of the fr2dBDA are described below.

\section{(c) Numerical solutions and interpretation}

Figure 6 shows the steady state solutions of the non-rotating $(\nu=0)$ fr2dBDA equations with $h=3 \mathrm{~cm}(M=12.5)$ at three different criticalities $\Gamma=0.208$, -0.625 and -1.458 corresponding to towing speeds $U=11.5,13.5$ and $15.5 \mathrm{~cm} \mathrm{~s}^{-1}$. The left panels show the linear solutions and the right panels the nonlinear solutions, allowing the effects of nonlinearity to be easily identified. At the subcritical towing speed $U=11.5 \mathrm{~cm} \mathrm{~s}^{-1}$, nonlinearity generates two weak bow waves up to $30 L$ ahead of the obstacle, with the wave pattern behind the obstacle modified only slightly from its linear counterpart. At weakly supercritical towing speeds (not shown) the two bow waves are stronger and form closer to the obstacle. With increasing supercriticality the inner bow wave decays, its curvature increases, and it eventually merges with the dispersive wave pattern. By moderate supercriticality $\left(U=13.5 \mathrm{~cm} \mathrm{~s}^{-1}\right.$ panel) there is, therefore, only a single, large-amplitude bow wave ahead of the obstacle. With further increases in criticality this solitary bow wave begins to decay and also merges with the dispersive wave pattern until by $U=15.5 \mathrm{~cm} \mathrm{~s}^{-1}$ the $\mathrm{V}$-wave pattern (e.g. Jiang \& Smith 2000) strongly resembles its linear counterpart. 
The numerical results of figure 6 can be compared directly with the experimental results shown in figure 5: both show similar developments in the forced wave pattern once allowance is made for the difference in the critical speed $c_{0}$ between the numerical results and the experiments caused inter alia by the diffusion acting on the stratification profile in the experiments. The two weak bow waves of the $U=7.5 \mathrm{~cm} \mathrm{~s}^{-1}$ experiment are stronger and closer to the obstacle in the $U=10.0 \mathrm{~cm} \mathrm{~s}^{-1}$ experiment, whereas in the $U=12.5 \mathrm{~cm} \mathrm{~s}^{-1}$ experiment only a single bow wave with much greater curvature remains distinct from the dispersive wave pattern behind the obstacle.

Figure 7 illustrates how increasing nonlinearity affects the wave pattern. The $h=3 \mathrm{~cm}(M=12.5), U=13.5 \mathrm{~cm} \mathrm{~s}^{-1}$ numerical solution (left panel) should be compared with its $h=5 \mathrm{~cm}(M=20.83)$ counterpart (right panel). Numerical integrations of the fKP equation in JV similarly show that increased nonlinearity, or equivalently reduced dispersion, leads to the formation of multiple solitary bow waves ahead of the obstacle. This transition between different solitary wave regimes has profound implications for the drag exerted by the obstacle on the flow (JV). Examining figure 7 and series of similar numerical solutions (not shown) shows that the extent to which the solitary wave pattern extends ahead of the obstacle depends only weakly on nonlinearity, but depends sensitively on the criticality, as can be seen in figure 6 .

Figure 8 shows linear and nonlinear rotating steady states for the numerical integrations with $f=0.105 \mathrm{~s}^{-1}$ (so $\nu=0.587$ ), corresponding to a Rossby radius of $c_{0} / f=1.3 \mathrm{~m}$. To emphasize the nonlinearity of the wave pattern topographic forcing has been chosen to be strong with $h=6 \mathrm{~cm}$ so $M=25$. Because of the increased nonlinearity, and the tendency of the nonlinear solutions in the rotating regime to form extremely sharp-crested waves (e.g. Shrira 1981, 1986; Grimshaw et al. 1998b; Esler et al. in press), it was found necessary to apply either a weak diffusion $\left(=-\kappa \nabla^{2} \eta_{0 \zeta}\right.$, where $\left.\nabla^{2} f=f_{\zeta \zeta}+f_{Y Y}\right)$ or hyper-diffusion $\left(=\kappa_{H} \nabla^{4} \eta_{0 \zeta}\right)$ to the right-hand side of equation (3.1) in order to maintain numerical stability. Very similar wave patterns were obtained with either choice, although the diffusion operator had a stronger damping effect on the wavetrain far behind the obstacle. Figure 8 was generated using hyper-diffusion with $\kappa_{H}=5.4 \times 10^{-4}$.

The subcritical $\left(U=11.5 \mathrm{~cm} \mathrm{~s}^{-1}\right)$ and moderately supercritical $\left(U=13.5 \mathrm{~cm} \mathrm{~s}^{-1}\right)$ numerical solutions show that the main effect of nonlinearity in rotating flow in this parameter regime occurs in the wake behind the obstacle. The bow wave is relatively unmodified by nonlinearity, but the trough following the obstacle (marked as $T$ in figure 5) is broadened and deepened and is followed by either one (for $U=13.5 \mathrm{~cm} \mathrm{~s}^{-1}$ ) or two (for $U=11.5 \mathrm{~cm} \mathrm{~s}^{-1}$ ) sharp-crested, large-amplitude solitary waves, which do not appear in the linear solutions. The crests of the solitary waves can be seen to merge with those of the dispersive wavetrain away from the centre axis. The dispersive wavetrain itself is modified by nonlinearity, as the waves have sharp crests and relatively broad troughs.

Interpreting the rotating, experimental results on the basis of figure 8 shows clearly, for $U=10 \mathrm{~cm} \mathrm{~s}^{-1}$, a bow wave (with crest $C_{1}$ in figures $4 b$ and 5 ) and a steep, large amplitude wave (with crest $C_{2}$ in figures $4 b$ and 5) following the broad deep trough after the obstacle (marked as $T$ in figure 5). As in the numerical solutions wave $C_{2}$ is a significant distance behind the obstacle (over $2 \mathrm{~m}$ ) and maintains its amplitude towards the centre axis. Similarly to 

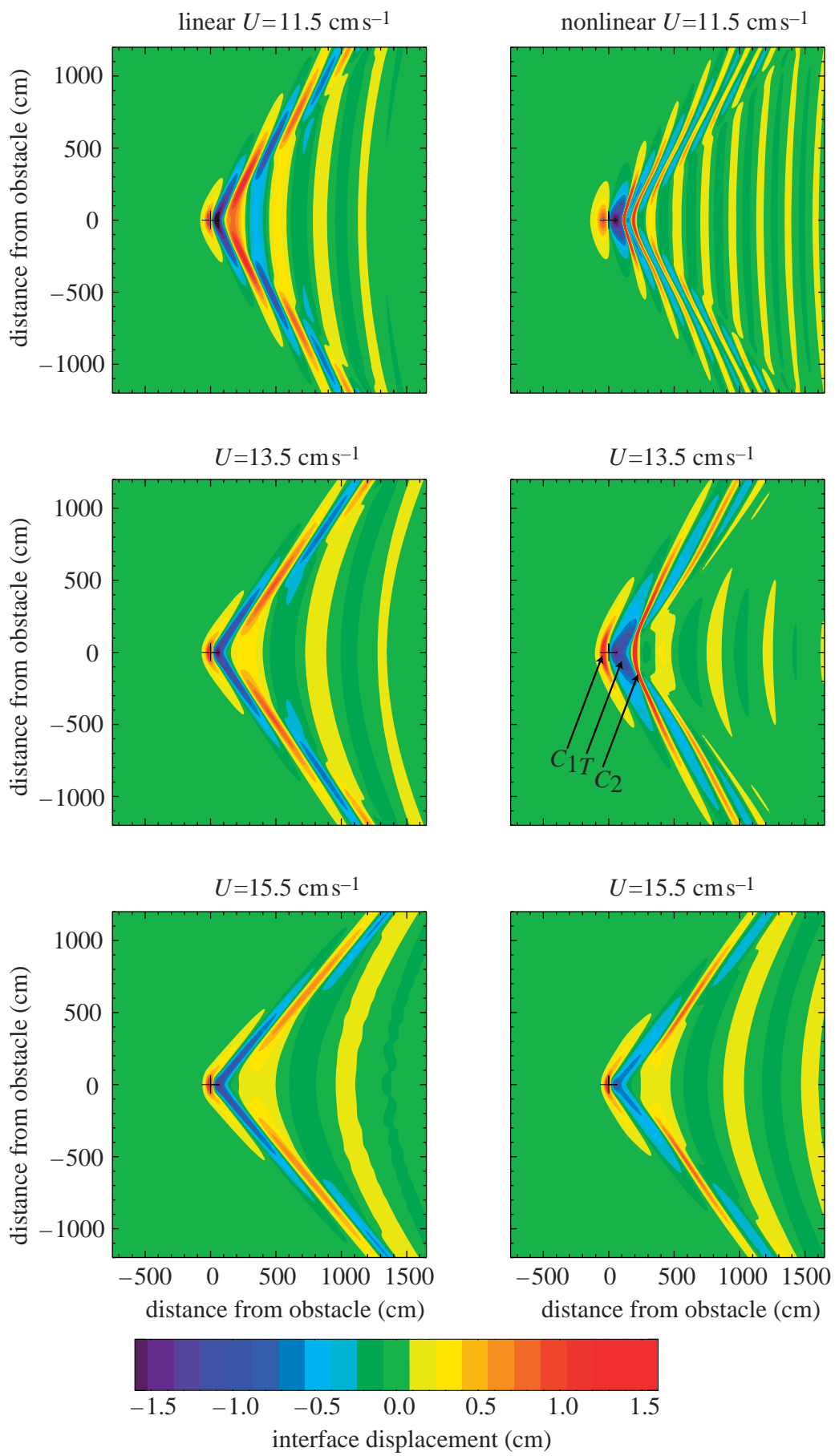

Figure 8. Linear and nonlinear rotating fr2dBDA steady states corresponding to obstacle height $h=6 \mathrm{~cm}(M=25)$ and towing speeds $U=11.5,13.5$ and $15.5 \mathrm{~cm} \mathrm{~s}^{-1}(\Gamma=0.208,-0.625,-1.458)$. As in the corresponding experiments, $C_{1}$ is the bow wave; $T$, the long, deep trough; and $C_{2}$, the sharp-crested lee wave in near-critical rotating flow. 


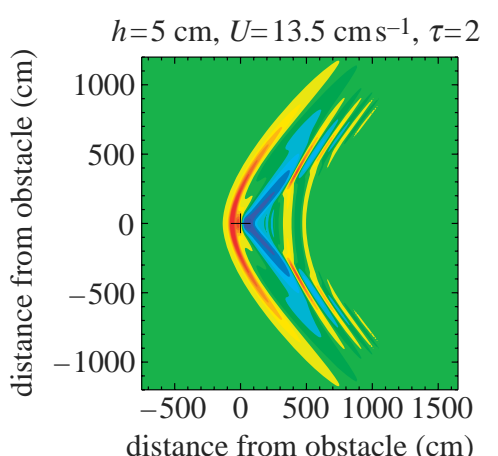

distance from obstacle $(\mathrm{cm})$
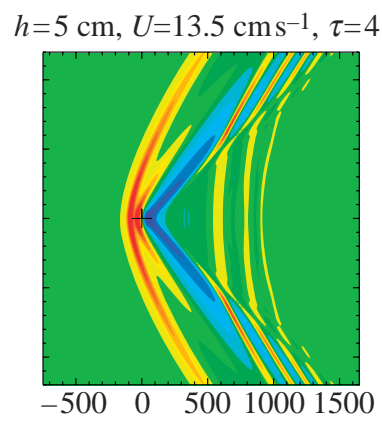

distance from obstacle $(\mathrm{cm})$ $h=5 \mathrm{~cm}, U=13.5 \mathrm{~cm} \mathrm{~s}^{-1}, \tau=8$

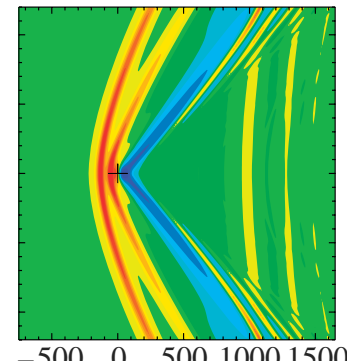

$\begin{array}{llrl}-500 & 0 & 50010001500\end{array}$

distance from obstacle $(\mathrm{cm})$

\begin{tabular}{ccccccc}
\hline-1.5 & -1.0 & -0.5 & 0.0 & 0.5 & 1.0 & 1.5 \\
& & & interface displacement $(\mathrm{cm})$ & &
\end{tabular}

Figure 9. Snapshots at $\tau=2,4$ and 8 illustrating the temporal development of the non-rotating f2dBDA numerical solution when $h=5 \mathrm{~cm}$ and $U=13.5 \mathrm{~cm} \mathrm{~s}^{-1}$.

Karpan (1967) for one -layer non-rotating flow, it can be shown that the far-field weakly nonlinear supercritical steady two-dimensional rotating flow is governed by the corresponding one-dimensional unsteady equation provided the crossstream coordinate of the two-dimensional equation is identified with the slow time of the one-dimensional equation. In Vilenski \& Johnson (2004) this allows solutions of the frKP equation to be interpreted in terms of the Ostrovsky (rotating KdV) equation (Ostrovsky 1978). Here a similar interpretation is possible and the cross-stream development of the interface displacement observations of figure $4 b$ is closely modelled by the temporal development of a solution of the one-dimensional unforced rotating BDA equation.

To our knowledge this is this first time this type of topographically forced nonlinear solitary wave has been recorded experimentally in a rotating fluid system. By contrast, it is debatable whether there is a clear signature of nonlinearity in the supercritical flow $\left(U=12.5 \mathrm{~cm} \mathrm{~s}^{-1}\right)$ or indeed, with the exception of the multiple bow waves, in the subcritical flow $\left(U=7.5 \mathrm{~cm} \mathrm{~s}^{-1}\right)$.

\section{Discussion and conclusions}

Experimental observations have been presented of the wavefield forced by a finite height obstacle travelling at near-critical speed through a two-layer rotating and non-rotating fluid in a tank sufficiently large to allow the lateral development of the pattern to be observed without interference from wall reflections. Comparisons with numerical solutions of the model fr2dBDA equation show that close to criticality bow waves appear in the experimental flows that are absent from linear theory but modelled by the weakly nonlinear equation. Further from criticality the difference between the linear and nonlinear flows disappears and the experimental results are well described by linear theory. Rotation is shown to introduce sharp crested highly nonlinear waves in the wake behind a near-critical obstacle. Recent exact solutions for fully nonlinear transcritical flow over a one-dimensional parabolic ridge in rotating shallow 
water (Esler et al. in press) typically show a shock downstream of the ridge followed by a wavetrain of finite-amplitude Poincare waves of limiting amplitude (Shrira 1981, 1986; Grimshaw et al. 1998b). Numerical integrations (not shown here) of a fully nonlinear, finite-volume, shallow water model show shocks appearing behind obstacles in non-dispersive rotating flows, providing further evidence of the nonlinearity of the sharp-crested waves of figure 5. The experiments of figure 5 also show that rotation confines the wake to within a distance of order the internal Rossby radius, $c_{0} / f$ for critical speed $c_{0}$ and Coriolis parameter $f$, of the axis. More rapid rotation confines the wake more closely to the axis and experiments for rotation periods of $T=60 \mathrm{~s}$ and $T=90 \mathrm{~s}$ ( $\mathrm{so} f=0.06$, $0.08 \mathrm{~s}^{-1}$ ), giving Rossby radii of 50 and $75 \mathrm{~cm}$, gave a clearly visible wake with little signature away from the axis and so not shown here.

The presentation of the experimental results as a fixed wave pattern travelling with the obstacle assumes that the flow has had sufficient time to become steady before the waves arrive at the probe spar. The obstacle reaches the spar in the middle of the tank in non-dimensional time in the range $\tau=3.8-6.4$, although waves forming the outer edges of the pattern can take twice as long to arrive. Some indication of the extent to which this is sufficient for nonlinear bow waves to develop may be gauged from figure 9 , which shows the temporal development of the bow waves in the case of the non-rotating multiple bow wave numerical solution with $h=5 \mathrm{~cm}, U=13.5 \mathrm{~cm} \mathrm{~s}^{-1}$. The steady state for this experiment is shown in figure 7 (right panel). Figure 9 shows the wavefield at $\tau=2,4$ and 8 . At $\tau=2$ a single bow wave has separated from the dispersive wake, while by $\tau=4$ a second bow wave has begun to separate. It is not until $\tau=8$, however, that the bow wave pattern begins to resemble the final state shown in figure 7 (right panel). It is clear, therefore, that although significant development of nonlinear bow waves has taken place by the time the wave pattern passes the probe spar in the experiments, it is by no means certain that the bow wave pattern has attained its steady state. Experiments intended to detect multiple bow wave patterns would thus need careful design to ensure that $\tau$ is sufficiently large when measurements are taken.

This work was funded by the UK Natural Environment Research Council Grant NER/A/S/2000/01323 and under the European Union contract HPRI-2001-CT-00168, 'Access to Research Infrastructures' of the program 'Improving Human Potential'. The authors are grateful to Henri Didelle and Samuel Viboud for their expert technical assistance.

\section{References}

Akylas, T. R. 1994 Three-dimensional long water-wave phenomena. Ann. Rev. Fluid Mech. 26, 191-210. (doi:10.1146/annurev.fl.26.010194.001203)

Benjamin, T. 1967 Internal waves of permanent form in fluids of great depth. J. Fluid Mech. 29, 559-592.

Burk, S. D. \& Haack, T. 1999 The dynamics of wave clouds upwind of coastal orography. Mon. Weather Rev. 28, 1438-1455.

Davis, R. E. \& Acrivos, A. 1967 Solitary internal waves in deep water. J. Fluid Mech. 29, 593-607.

Durran, D. R. 2000 Small-amplitude coastally trapped disturbances and the reduced-gravity shallow-water approximation. Q. J. R. Meteorol. Soc. 126, 2671-2689. (doi:10.1256/smsqj. 56903) 
Ertekin, W. C., Webster, R. C. \& Wehausen, J. V. 1986 Waves caused by moving disturbance in a shallow channel of finite width. J. Fluid Mech. 169, 275-292.

Esler, J. G., Rump, O. J., Johnson, E. R. In press. Steady rotating flows over a ridge. Phys. Fluids.

Grimshaw, R. 1985 Evolution-equations for weakly nonlinear, long internal waves in a rotating fluid. Stud. Appl. Math. 73, 1-33.

Grimshaw, R. H. J. \& Smyth, N. 1986 Resonant flow of a stratified fluid over topography. J. Fluid Mech. 169, 429-464.

Grimshaw, R. H. J., He, J. M. \& Ostrovsky, L. A. 1998a Terminal damping of a solitary wave due to radiation in rotational systems. Stud. Appl. Math. 101, 197-210. (doi:10.1111/1467-9590. 00090)

Grimshaw, R. H. J., Ostrovsky, L. A., Shrira, V. I. \& Stepanyants, Y. A. 1998 b Long nonlinear surface and internal gravity waves in a rotating ocean. Surv. Geophys. 19, 289-338. (doi:10. 1023/A:1006587919935)

Grimshaw, R. H. J., Chan, K. H. \& Chow, K. W. 2002 Transcritical flow of a stratified fluid: The forced extended Korteweg-de Vries model. Phys. Fluids 14, 755-774. (doi:10.1063/1.1429962)

Jiang, Q. \& Smith, R. B. 2000 V-waves, bow shocks, and wakes in supercritical hydrostatic flow. J. Fluid Mech. 406, 27-53. (doi:10.1017/S0022112099007636)

Johnson, R. S. 1997 A modern introduction to the mathematical theory of water waves. Cambridge: Cambridge University Press.

Johnson, E. R. \& Vilenski, G. G. 2004 Flow patterns and drag in near-critical flow over isolated orography. J. Atmos. Sci. 61, 2909-2918. (doi:10.1175/JAS-3311.1)

Johnson, E. R., Vilenski, G. G. 2005 Two-dimensional leaps in near-critical flow over isolated orography. Proc. R. Soc. A. (doi:10.1098/rspa.2005.1530)

Kadomtsev, B. B. \& Petviashvili, V. I. 1970 On the stability of solitary waves in weakly dispersing media. Sov. Phys. Dokl. 15, 539-541.

Karpman, V. I. 1967 The structure of two-dimensional flow around bodies in dispersive media. JETP 23, 1102-1111.

Lee, S. J. \& Grimshaw, R. H. J. 1990 Upstream-advancing waves generated by 3-dimensional moving disturbances. Phys. Fluid A-Fluid Dyn. 2, 194-201. (doi:10.1063/1.857769)

Li, Y. \& Sclavounos, P. D. 2002 Three-dimensional nonlinear solitary waves in shallow water generated by an advancing disturbance. J. Fluid Mech. 470, 383-410. (doi:10.1017/ S0022112002001568)

Li, X. F., Dong, C. M., Clemente-Colon, P., Pichel, W. G. \& Friedman, K. S. 2004 Synthetic aperture radar observation of the sea surface imprints of upstream atmospheric solitons generated by flow impeded by an island. J. Geophys. Res. Oceans 109, C02016.

Lighthill, M. J. 1965 Group velocity. J. Inst. Math. Appl. 1, 1-28.

Matsuno, Y. 1995 Forced Benjamin-Ono-equation and its application to soliton dynamics. Phys. Rev. E 52, 6333-6343. (doi:10.1103/PhysRevE.52.6333)

Maxworthy, T., Chabert, G., Dhieres, D. \& Didelle, H. 1984 The generation and propagation of internal gravity-waves in a rotating fluid. J. Geophys. Res. Oceans 89, 6383-6396.

Melville, W. K. \& Helfrich, K. R. 1987 Transcritical two-layer flow over topography. J. Fluid Mech. 178, 31-52.

Ono, H. 1975 Algebraic solitary waves in stratified fluids. J. Phys. Soc. Jpn 39, 1083-1091.

Ostrovsky, L. A. 1978 Non-linear internal waves in a rotating ocean. Okeanologiya 18, 181-191.

Ramirez, C. \& Renouard, D. 1998 Generation of internal waves over a shelf. Dyn. Atmos. Oceans 28, 107-125. (doi:10.1016/S0377-0265(98)00048-7)

Rottman, J. W. \& Einaudi, F. 1993 Solitary waves in the atmosphere. J. Atmos. Sci. 50, 2116-2136. (doi:10.1175/1520-0469(1993)050<2116:SWITA > 2.0.CO;2)

Samelson, R. M. 1992 Supercritical marine-layer flow along a smoothly varying coastline. J. Atmos. Sci. 49, 1571-1584. (doi:10.1175/1520-0469(1992)049<1571:SMLFAA > 2.0.CO;2)

Samelson, R. M. 1999 The vertical structure of linear coastal-trapped disturbances. Mon. Weather Rev. 127, 201-213. (doi:10.1175/1520-0493(1999)127<0201:TVSOLC > 2.0.CO;2) 
Samelson, R. M. \& Lentz, S. J. 1994 The horizontal momentum balance in the marine atmospheric boundary-layer during CODE-2. J. Atmos. Sci. 51, 3745-3757. (doi:10.1175/15200469(1994)051<3745:THMBIT > 2.0.CO;2)

Schär, C. \& Smith, R. B. 1993 Shallow-water flow past isolated topography. 2. Transition to vortex shedding. J. Atmos. Sci. 50, 1401-1412.

Schmidt, G. A. \& Johnson, E. R. 1993 a Direct calculation of low-frequency coastally trapped waves and their scattering. J. Atmos. Ocean. Technol. 10, 368-380. (doi:10.1175/15200426(1993)010<0368:DCOLFC > 2.0.CO;2)

Schmidt, G. A. \& Johnson, E. R. $1993 b$ Grid dependence in the numerical determination of topographic waves. Ocean Modelling 98, 10-11.

Shrira, V. I. 1981 Propagation of long nonlinear waves in a layer of rotating fluid. Izvestiya Atmos. Ocean. Phys. 17, 55-59.

Shrira, V. I. 1986 On long strongly nonlinear waves in a rotating ocean. Izvestiya Atmos. Ocean. Phys. 22, 298-305.

Soderberg, S. \& Tjernstrom, M. 2001 Supercritical channel flow in the coastal atmospheric boundary layer: idealized numerical simulations. J. Geophys. Res. Atmos. 106, $17811-17829$. (doi:10.1029/2001JD900195)

Stevenson, R. E. 1969 The 200-mile fishline. In Oceans from space (ed. P. C. Badgley, L. Miloy \& L. F. Childs). Houston: Gulf Publishing Company.

Tjernstrom, M. \& Grisogono, B. 2000 Simulations of supercritical flow around points and capes in a coastal atmosphere. J. Atmos. Sci. 57, 108-135.

Vilenski, G. G. \& Johnson, E. R. 2004 Near-critical free-surface rotating flow over topography. Proc. R. Soc. A 460, 2865-2881. (doi:10.1098/rspa.2004.1317) 